\title{
Rapeseed Oil in New Application: Assessment of Structure of Oleogels Based on their Physicochemical Properties and Microscopic Observations
}

\author{
Milena Kupiec ${ }^{1}\left(\mathbb{D}\right.$, Anna Zbikowska ${ }^{1} \mathbb{D}$, Katarzyna Marciniak-Lukasiak ${ }^{1, * \mathbb{D}}$ \\ and Małgorzata Kowalska 2 (D) \\ 1 Institute of Food Sciences, Faculty of Food Assessment and Technology, Warsaw University of Life \\ Sciences (WULS-SGGW), Nowoursynowska st. 159c, 02-776 Warsaw, Poland; \\ milena_kupiec@sggw.edu.pl (M.K.); anna_zbikowska@sggw.edu.pl (A.Z.) \\ 2 Faculty of Chemical Engineering and Commodity Science, Kazimierz Pulaski University of Technology \\ and Humanities, Chrobrego st. 27, 26-600 Radom, Poland; mkowalska7@vp.pl \\ * Correspondence: katarzyna_marciniak_lukasiak@sggw.edu.pl; Tel.: +48-22-59-37548
}

Received: 7 May 2020; Accepted: 5 June 2020; Published: 8 June 2020

\begin{abstract}
The aim of the study was to analyze the effect of the type of gelling substance on the selected physicochemical properties of oleogels. Rapeseed oil (RO) and 5\% w/w of one of the following structuring compounds (SC) were used for their production waxes: sunflower (SUN), candelilla (CAN), bees white (BW), bees yellow (BY), or monoacylglycerols (MAG). The ability of SC to form a network in $\mathrm{RO}$ was assessed on the basis of an analysis of the texture of oleogels (hardness and spreadability test, stability determined by the centrifugal method). Oxidative stability was determined using the Rancimat test. In addition, the microstructure of an oleogel samples was observed at a magnification of 600 times in polarized light and in a bright field. Differences in the brightness and color of the samples were also assessed using the CIELab reflection method. The highest hardness (5.52 N) and physical stability (99.67\%) were found in oleogel with candelilla wax. A higher value of the force causing deformation of the sample indicates a denser network of oil gel, which was confirmed by the microscopic images analyzed in the work. The organogel with CAN had the shortest (on average $8.49 \mathrm{~nm}$ ) and the most regularly distributed crystals compared to other samples. The lowest values of organogel strength and spreadability test parameters (e.g., firmness, work of shear, stickness, work of adhesion), which were respectively $0.73 \mathrm{~N}, 4.39 \mathrm{~N}$, and $9.74 \mathrm{~N} \mathrm{~mm}$, while $-4.87 \mathrm{~N}$ and $-2.68 \mathrm{~N}$ mm were obtained with the variant, which was yellow beeswax. Considering the texture results obtained and the centrifugal stability, it was found that organogels with yellow beeswax were characterized by the worst structuring of rapeseed oil. Sunflower wax was considered the best among the analyzed gelling agents (under the conditions of the experiment). The organogel with its 5\% $w / w$ share was distinguished by the closest to the white color and texture most reminiscent of cream. In addition, it had the highest work of shear value and the longest induction time $(6.8 \mathrm{~h})$ in the Rancimat test.
\end{abstract}

Keywords: rapeseed oil; gelling agents; oleogelation; oleogel; waxes

\section{Introduction}

Rapeseed is the second major oily plant grown in the world (after soybean). Its cultivation is estimated at over 68 million metric tons [1]. The biggest world production in the 2019/2020 season took place in Canada (19,000 metric tons), European Union countries (17,000 metric tons) and in China $(13,100$ metric tons) [1]. It owes its popularity to, among others, high fat (above $41 \%)$, but also protein (about 40\%) [2]. Therefore, rapeseed is a valuable raw material not only in the production of edible 
oils, biodiesel, or animal feed (Low Erucic Acid Rapeseed, LEAR), but also paints, lubricants, or cling film and polythene products (High Erucic Acid Rapeseed, HEAR) [3]. Other potential uses of rapeseed include, for example, the extraction of protein hydrolysates and their incorporation in functional foods [4].

The most traditional product obtained from LEAR rapeseed oil (RO) is recommended as a fat component of the human diet due to the high content of monounsaturated fatty acids and acids from the n-3 group, among other reasons [5]. The oleic acid contained in $\mathrm{RO}$ reduces the risk of cardiovascular disease, including heart attack, by lowering blood pressure [6] and lowering total cholesterol [7]. The Food and Drug Administration (FDA) approved a health claim for rapeseed oil, suggesting that it may help reduce the risk of cardiovascular disease if saturated fat will be replaced by $\mathrm{RO}$ in the daily diet [8]. In addition, rapeseed oil is readily available, and its global production in 2019/2020 was above 27 million metric tons [9]. The largest RO production takes place in the European Union countries-9.39 million tons, China-5.93, Canada-4.25 and India-2.66 [1].

One of the new directions in the processing of rapeseed oil is oleogelation. It is a process that involves structuring liquid fats using gelling agents [10]. As a result, oils obtain a semi-solid/solid consistency, and their high nutritional value is maintained [11]. Gelling agents are usually systems containing fatty acid and fatty alcohol (e.g., stearyl alcohol and stearic acid mixture, behenyl alcohol and behenic acid mixture) [12], and their derivatives (e.g., thioester derivative) [13]. There are numerous scientific reports proving the solidifying properties of many chemicals, e.g., monoacylglycerols, polysaccharides, plant and animal waxes [14-17]. Waxes, e.g., rice and sunflower, are a waste product during the production of vegetable oils [18,19]. For sunflower oil, this is over 42.000 metric tons of wax [18], which can be used, e.g., as a structuring substance in the production of oleogels.

The use of liquid oils (e.g., rapeseed) in the form of oleogels has both health and technological benefits $[20,21]$. Their incorporation into food products may prevent or inhibit undesirable processes, e.g., the migration of cocoa butter to the chocolate surface [22], but also to increase the nutritional value of many products, e.g., cakes, cookies, and delicatessen products [23-26]. Oleogels can also be used in the cosmetics and pharmaceutical industries [27], or in the petrochemical industry, in which, as a mixture of gelling compounds and inflammatory substances, they would prevent their leakage [28]. This would have a direct impact on the increased safety of their storage and transport [29].

Vegetable oil gelling agents such as candelilla wax, sunflower wax, white beeswax, yellow beeswax, and monoacylglycerols are presented in many scientific publications as promising, with wide application possibilities. Despite numerous scientific publications, in which the possibility of obtaining oleogels with various gelling substances has been demonstrated, there is no direct work comparing the properties of oleogels obtained from rapeseed oil as well as waxes and monoacylglycerols used in equal concentrations. Therefore, in this study, the degree of structuring of RO particles by waxes such as candelilla, sunflower, beeswax yellow, beeswax white, and monoacylglycerols at $5 \% w / w$ concentration was assessed.

\section{Materials and Methods}

\subsection{Materials}

The experimental material consisted of oleogels that were produced with the participation of $95 \mathrm{~g}$ of rapeseed oil ("Kujawski-ZT" Kruszwica "S.A.) and $5 \mathrm{~g}$ of the following structuring agent. The following gelling agents were used: white (BW) and yellow (BY) beeswax (CAN; Norevo GmbH, Germany), candelilla wax (Norevo GmbH, Germany), sunflower wax (SUN; Kahl GmbH \& Co. KG, Germany) and monoacylglycerols (MAG; Verol N-90, Lasenor, Emul, Spain). The minimum concentration of structuring substances ( $\mathrm{cp}$ ) in the oil solvent, determined on the basis of scientific literature and preliminary research, was as follows: for the variant with SUN 1\%, CAN 2\%, BW and BY $3 \%$ [30-32]. In the case of monoacylglycerols, the formation of the gel structure in the entire sample volume was noted at a concentration of minimum $5 \% w / w$. At lower $\mathrm{cp}$, only the surface of the mixture 
was structured, under which the system remained semi-liquid. Based on the above data, the $5 \% w / w$ addition of the gelling agent was considered the lowest, ensuring the gel consistency of rapeseed oil in all tested variants. The physicochemical characteristics of the gelling substances are presented in Table 1.

Table 1. Specification of structuring agents [manufacturer's data].

\begin{tabular}{|c|c|c|c|c|c|}
\hline \multirow{2}{*}{$\begin{array}{c}\text { KERRYPNX } \\
\text { Parameter }\end{array}$} & \multicolumn{5}{|c|}{ Structuring Agent } \\
\hline & Beeswax White & Beeswax Yellow & Candelilla Wax & Sunflower Wax & Monoacylglycerols \\
\hline $\begin{array}{c}\text { Melting } \\
\text { temperature }\left[{ }^{\circ} \mathrm{C}\right]\end{array}$ & \multicolumn{2}{|c|}{$61-66$} & $68.50-72.50$ & $74-80$ & 63 \\
\hline Color & White & yellow & yellow & pale-yellowish & white-ivory \\
\hline $\begin{array}{l}\text { Acid value } \\
{[\mathrm{mg} \mathrm{KOH} / \mathrm{g}]}\end{array}$ & $17-24$ & $17-22$ & $12-22$ & $2-8$ & 1.40 \\
\hline $\begin{array}{l}\text { Ester value } \\
{[\mathrm{mg} \mathrm{KOH} / \mathrm{g}]}\end{array}$ & $70-80$ & $70-80$ & - & $67-93$ & - \\
\hline
\end{tabular}

\subsection{Oleogels Preparation}

The technology of producing oleogels was based on the initial liquefaction of gelling substances in rapeseed oil at $80^{\circ} \mathrm{C}$ and further heating $\left(90^{\circ} \mathrm{C}\right)$ while mixing. The total duration of the heating process, $20 \mathrm{~min}$, provided a clear solution, which was then allowed to solidify in a thermostatic cabinet (POL-EKO-APARATURA Sp. J., Type: ST 2/2 Basic, Poland) for $24 \mathrm{~h}$ at $20^{\circ} \mathrm{C}$. Three replicates of each of the five oleogel variants were performed.

\subsection{Methods}

\subsubsection{Test for Strength and Spreadability of Oleogels at $20 \pm 3{ }^{\circ} \mathrm{C}$}

Strength and spreadability tests were performed using a TX.AT plus device (Micro Stable Systems, Godalming, Surrey, UK) at room temperature. Oleogel hardness was determined by the penetration test using the $0.5 / \mathrm{P}$ tip. The samples were placed in cylindrical vessels with a capacity of $50 \mathrm{~mL}$ and a height of $2.5 \mathrm{~cm}$. The speed, at which the working element was lowered to a depth of $5 \mathrm{~mm}$, was $1 \mathrm{~mm} / \mathrm{s}$. Oleogel strength was the highest point on the strength chart [N] versus time [s].

The spreadability test consisted of immersing the upper working element (UWE, in the shape of an inverted cone) with a diameter of $4.5 \mathrm{~cm}$ in a special container, which was filled with the test sample and ideally suited to UWE. The speed at which the upper working element was lowered to get a $1 \mathrm{~mm}$ gap between it and container was $3 \mathrm{~mm} / \mathrm{s}$. The penetration distance was $25 \mathrm{~mm}$. The test results were obtained in the form of a force dependence curve $[\mathrm{N}]$ and time [s]. Firmness was determined as the highest value on the graph $[\mathrm{N}]$, while work of shear was determined as the area below the surface of the force-time graph $[\mathrm{N} \cdot \mathrm{mm}]$, stickness was determined as the point with the lowest value $[\mathrm{N}]$, and work of adhesion was determined as the area over the force-time curve [N.mm]. Nine measurements were made for each variant (strength and spreadability tests).

\subsubsection{Stability of Oleogel-Centrifuge Method}

Oleogel stability was determined based on the modified method of Da Pieve et al. [33]. Oil gel samples of $3 \pm 0.10 \mathrm{~g}$ were centrifuged in the MPW-352 at $4500 \mathrm{rpm}$ (Poland). The duration of the process was $10 \mathrm{~min}$. Then, the whole was weighed $\left(\mathrm{M}_{\mathrm{pw}}\right)$. After removing the oil, collected in the upper part of the sample, the residue weight $\left(\mathrm{M}_{\mathrm{g}}\right)$ was determined. The study was performed in triplicate for all variants.

Oleogel stability, determined by the centrifugal method (SO) was calculated based on the formula [34,35]:

$$
\mathrm{SO}=\left(\mathrm{M}_{\mathrm{g}}-\mathrm{M}_{\mathrm{p}}\right) /\left(\mathrm{M}_{\mathrm{pw}}-\mathrm{M}_{\mathrm{p}}\right) 100[\%]
$$

in which: $\mathrm{M}_{\mathrm{g}}$-tube and sample mass after centrifugation and removal of the liquid fraction [g]; $\mathrm{M}_{\mathrm{p}}$-tube weight [g]; $\mathrm{M}_{\mathrm{pw}}$-tube and sample mass after centrifugation [g]. 


\subsubsection{Color}

The color determination was carried out using the CIELab system (Konica-Minolta 200, Tokyo, Japan). The method is based on the representation of color, using three points $L^{*}, a^{*}, b^{*}$, which form a spatial solid [36]. Oleogels were placed in a vessel with a diameter of $14 \mathrm{~cm}$ and a height of about $5 \mathrm{~cm}$. Numerical values of the $L^{*}$ sample brightness, as well as parameters $a^{*}$ and $b^{*}$, were obtained indicating the color of oleogels. The first, one- $\mathrm{a}^{*}$, determines the share of green and red, while the second, one- $b^{*}$, determines the share of yellow and blue. The data were also used to determine the absolute color difference $\Delta \mathrm{E}$. Due to the lack of a control sample, a white standard was used to determine the differences between the color variants. For this purpose, the following formula was used [37]:

$$
\Delta E=\sqrt{\left(L_{p}^{*}-L_{x}^{*}\right)^{2}+\left(a_{p}^{*}-a_{x}^{*}\right)^{2}+\left(b_{p}^{*}-b_{x}^{*}\right)^{2}}
$$

in which the control test was a standard white calibration plate with the following parameters: $L_{p}^{*}=97.83, a_{p}^{*}=-0.45$, and $b_{p}^{*}=1.88$. Numerical values obtained for a specific oleogel variant were substituted for the symbols with the $x$ tag.

\subsubsection{Oxidation Stability Index: The Rancimat Method}

Determination of the oxidative stability of oleogels by an accelerated oxidation test was performed on a Rancimat type 679 apparatus (Metrohm, Herisau, Switzerland). During the test, the fat sample is exposed to air and high temperature in a closed circuit. The air flowing through the sample transfers its volatile oxidation products into a measuring vessel containing demineralized water. A sudden, significant increase in measured conductivity in the vessel indicates the induction time [h]. In a Rancimat test of oleogels, the following parameters were used: a sample weight of $2.5 \mathrm{~g}$, a temperature of $120^{\circ} \mathrm{C}$, air flow of $20 \mathrm{~L} / \mathrm{h}$, volume of water in a conductivity vessel of $60 \mathrm{~mL}$. The test was carried out in triplicate.

\subsubsection{Analysis of Oleogel Morphology}

Observation of organogel fat crystals was carried out using a biological microscope (Delta Optical 100 TP, Mińsk Mazowiecki, Poland) using two microscopic techniques: bright field and simple polarization. Pictures of samples at room temperature $\left(20 \pm 3^{\circ} \mathrm{C}\right)$ were taken using a microscope camera (Delta Optical DLT CAM PRO, Mińsk Mazowiecki, Poland). Observations were made at 600 times magnification. The length of fat crystals was determined for 27 measurements for each variant.

\subsubsection{Statistical Analysis}

Statistical analysis and data plots were performed using the Statistica 13 program. Data on the normal distribution were analyzed using a one-way ANOVA variance analysis. The significance of differences between means was determined based on the Tukey test, at the level of significance $\alpha=0.05$. Non-parametric data (fatty crystal length) were analyzed using the Kruskal-Wallis rank test.

\section{Results and Discussion}

\subsection{Oleogel Strengh and Spreadability Test}

Samples of rapeseed oleogels were characterized by various physical properties, including strength (Table 2). The maximum force of deformation was obtained for an oil gel with candelilla wax (CAN) $5.52 \mathrm{~N}$. This is more than seven times the value of this parameter, compared to oleogels with beeswax yellow (BY) and monoacylgycerols (MAG) (Table 2). In the case of the spreadability test, the highest firmness and work of shear values were obtained for oleogels containing SUN and CAN. These samples did not differ statistically significantly (at $\alpha=0.05$ ). This indicates the greatest strength needed to spread 
them. The lowest values of stickness $(-20.37 \mathrm{~N})$ and work of adhesion $(-7.54 \mathrm{~N} \mathrm{~mm})$ were recorded for organogel with sunflower wax.

Table 2. The results of oleogel strengh test (first column) and spreadability test of oleogels (firmness, work of shear, stickness, and work of adhesion).

\begin{tabular}{cccccc}
\hline Variant $^{*}$ & Oleogel Strengh [N] & Firmness [N] & Work of Shear [N·mm] & Stickness [N] & Work of Adhesion [N·mm] \\
\hline CAN & $5.52 \pm 0.33 \mathrm{~d}$ & $12.68 \pm 1.06 \mathrm{~b}$ & $35.57 \pm 3.88 \mathrm{~b}$ & $-10.06 \pm 0.83 \mathrm{a}$ & $-5.01 \pm 0,48 \mathrm{~d}$ \\
MAG & $0.77 \pm 0.15 \mathrm{a}$ & $6.47 \pm 0.46 \mathrm{a}$ & $18.29 \pm 1.34 \mathrm{a}$ & $-7.50 \pm 0.31 \mathrm{a}$ & $-3,81 \pm 0,42 \mathrm{a}$ \\
BW & $1.60 \pm 0.31 \mathrm{~b}$ & $5.60 \pm 0.42 \mathrm{a}$ & $13.00 \pm 1.05 \mathrm{a}$ & $-6.22 \pm 0.39 \mathrm{a}$ & $-2.92 \pm 0.18 \mathrm{a}, \mathrm{b}$ \\
BY & $0.73 \pm 0.15 \mathrm{a}$ & $4.39 \pm 0.42 \mathrm{a}$ & $9.74 \pm 1.44 \mathrm{a}$ & $-4.87 \pm 0.41 \mathrm{a}$ & $-2.68 \pm 0.34 \mathrm{~b}$ \\
SUN & $3.33 \pm 0.42 \mathrm{c}$ & $15.78 \pm 3.12 \mathrm{~b}$ & $46.62 \pm 8.60 \mathrm{~b}$ & $-20.37 \pm 4.53 \mathrm{a}$ & $-7.54 \pm 0.26 \mathrm{c}$ \\
\hline
\end{tabular}

* CAN—oleogel with candelilla wax; MAG—oleogel with monoacylglycerols; BW—oleogel with white beeswax; BY—oleogel with yellow beeswax; SUN—oleogel with sunflower wax; a,b,c,d-the same letters at the same column indicate that there is no statistical difference (at $\alpha=0.05$ ).

Yllmaz and Öğütcü [34] for oleogels containing 7\% w/w monoacylglycerols in hazelnut oil (HO) obtained a firmness of $0.82 \mathrm{~N}$ at room temperature. This value is much lower than that achieved by the MAG variant with a lower concentration $(5 \% w / w)$ in rapeseed oil (Table 2). The reason for the differences in the values of the mentioned oleogel parameter is probably a different type of fatty solvent, due to which MAG, as a gelling substance has different structuring properties. The differences may also result from different values of room temperature, at which the determination was carried out. Yılmaz and Öğütcü [34] also obtained lower firmness for HO-based oleogels, with a 7\% w/w MAG content, compared to all other rapeseed oil gels (CAN, BW, BY, SUN).

\subsection{Oleogel Stability, Determined by the Centrifugal Method}

In order to check the durability of rapeseed oil binding by gelling agents, a centrifugal test was carried out. The organogels set in rotation were separated into two phases: liquid and solid-gel. The obtained results testify to the relatively high stability of the oleogel structures, which were obtained with a $5 \%$ addition of various structure-forming substances in rapeseed oil (Figure 1). Among the tested oleogels, candelilla wax and white beeswax samples proved to be the most durable systems for centrifugal force. They achieved stability of $99.67 \%$ for CAN and $97.80 \%$ for BW (Figure 1). Oleogel with BY had the largest leakage of the oil phase after centrifugation $(89.30 \%)$. In turn, variants with SUN and MAG achieved stability that was not statistically different (at $\alpha=0.05$ ) from other organogels (Figure 1). Da Pieve et al. [33] for oleogel that was produced with $5 \% w / w$ monoacylglycerols, but in another solvent-cod liver oil-obtained about $20 \%$ loss of liquid fraction in the centrifuge test. The reason for the better stability of the MAG oleogel and the other structuring substances tested in rapeseed oil is the more than twice higher rotation speed used in the test by scientists $(10,000 \mathrm{rpm})$ and 5 min longer time of the test.

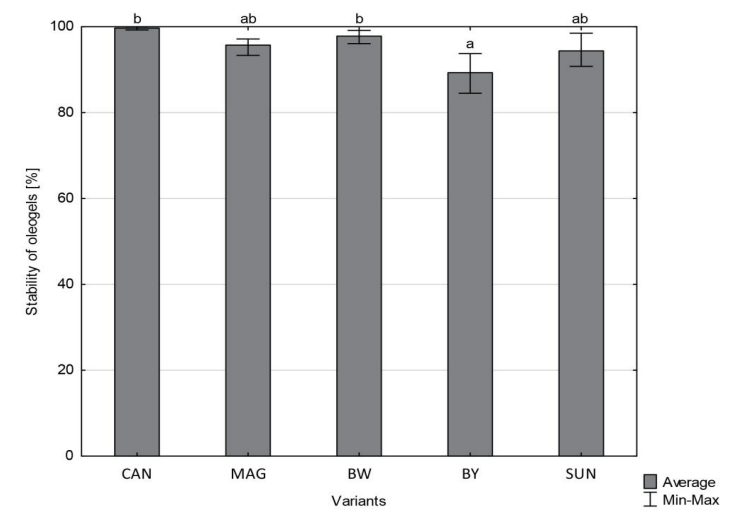

Figure 1. Share of unchanged oleogel structure after centrifugation, depending on the type of structure-forming substance: $\mathrm{a}, \mathrm{b}$ - the same letters above bars indicate that there is no statistical difference $(\alpha=0.05)$. 


\subsection{Oleogels Color}

The applied structure-forming substances caused statistically significant $(p \leq 0.05)$ color differentiation of the obtained oleogels (Table 3).

Table 3. The results of the determination of the color of oleogels, determined by three parameters: $\mathrm{L}^{*}$, $\mathrm{a}^{*}, \mathrm{~b}^{*}$ and absolute color difference $\Delta \mathrm{E}$.

\begin{tabular}{ccccc}
\hline \multicolumn{5}{c}{ Parameter } \\
\hline Variant of Oleogel & $\mathbf{L}^{*}$ & $\mathbf{a}^{*}$ & $\mathbf{b}^{*}$ & $\Delta \mathbf{E}$ \\
\hline CAN & $45.06 \pm 0.21 \mathrm{~b}$ & $-3.11 \pm 0.02 \mathrm{a}$ & $-2.70 \pm 0.02 \mathrm{~b}$ & $53.04 \pm 0.21 \mathrm{~d}$ \\
MAG & $47.99 \pm 0.19 \mathrm{c}$ & $-2.58 \pm 0.03 \mathrm{~b}$ & $-3.25 \pm 0.21 \mathrm{a}, \mathrm{b}$ & $50.15 \pm 0.21 \mathrm{~b}$ \\
BW & $46.04 \pm 0.09 \mathrm{a}$ & $-2.19 \pm 0.18 \mathrm{c}$ & $-3.41 \pm 0.37 \mathrm{a}$ & $52.09 \pm 0.08 \mathrm{c}$ \\
BY & $46.26 \pm 0.30 \mathrm{a}$ & $-3.02 \pm 0.04 \mathrm{a}$ & $3.09 \pm 0.18 \mathrm{~d}$ & $51.65 \pm 0.30 \mathrm{c}$ \\
SUN & $55.68 \pm 0.54 \mathrm{~d}$ & $-2.39 \pm 0.07 \mathrm{~b}, \mathrm{c}$ & $2.09 \pm 0.05 \mathrm{c}$ & $42.20 \pm 0.54 \mathrm{a}$ \\
\hline
\end{tabular}

The oleogel with sunflower wax achieved the highest $L^{*}$ parameter value, 55.68 (Table 3), which indicates its highest brightness. In turn, the darkest turned out to be candelilla wax organogel (45.06). Yılmaz and Öğütcü [34] for oleogels containing 3\%,7\%, and 10\% addition of a gelling substance in the form of monoacylglycerols in hazelnut oil obtained the values of the parameter $L^{*}$ (respectively): $42.85,47.96$, and 54.52. This suggests that as the amount of MAG increases, the brightness of the oleogel color increases. A very similar $L^{*}(0.02$ higher $)$ was achieved by an organogel containing the same gelling substance, but in a lower concentration $(5 \% w / w)$ and in a different solvent (rapeseed oil-OR) (Table 3). This is confirmation that OR has a lighter color than hazelnut oil, which also affects the final color of the oil gels.

For all oleogels, the values of parameter $\mathrm{a}^{*}$ were negative, which indicates a greater proportion of green hue (Table 3$)$. The least intensity of this color was characterized by organogels with beeswax white (-2.19), sunflower wax (-2.39), and monoacylglycerols $(-2.58)$. Variants with CAN and BY had the largest share of green. Similar results (negative values of parameter $\mathrm{a}^{*}$ ) were also obtained by other authors for organogels consisting of monoacylglycerols $(3 \%, 7 \%$, or $10 \% w / w)$ and hazelnut oil. The value of parameter $a^{*}$ for oleogel with $7 \%$ of MAG was -2.42 [34]. This value is 0.16 units smaller than that obtained for the variant with the same gelling substance but with a lower concentration and a different lipid solvent (Table 3).

The $b^{*}$ coordinate reached a positive value in SUN and BY oleogels, which indicates a greater proportion of yellow. Other variants reached values below zero, which indicates a greater proportion of blue. The lowest value among the tested organogels was achieved by the variant with white beeswax, which proves its most cold shade. It is also a confirmation that the yellow shade of the oleogels is affected not only by the color of the fatty solvent, but also by the gelling substance dispersed in it.

Analysis of the xyz chart of the dependence of the $L^{*}$ color parameters on $a^{*}$ and $b^{*}$ obtained by all rapeseed oleogels shows that the brightest color was reached by variants with $\mathrm{a}^{*}$ values in the range from -2.7 to -2.3 and $b^{*}$ from 0 to 2 (Figure 2).

In all tested organogels, the absolute color difference was above 40 (Table 3). According to Onacik-Gür and Żbikowska [26], if the value of $\Delta \mathrm{E}$ is greater than three, then this difference is observable by the human eye. Therefore, taking into account the visual assessment, all tested variants differed significantly from the standard whiteness. The lowest average $\Delta \mathrm{E}$ value, equal to 42.20 , was found in oleogel containing sunflower wax. Therefore, its color was the closest in terms of parameters $\mathrm{L}^{*}, \mathrm{a}^{*}$, and $\mathrm{b}^{*}$ to the white standard. In the case of organogels with candelilla wax, the $\Delta \mathrm{E}$ value is the highest and amounts to 53.04. This variant differed from the whiteness standard more than other oleogels. 


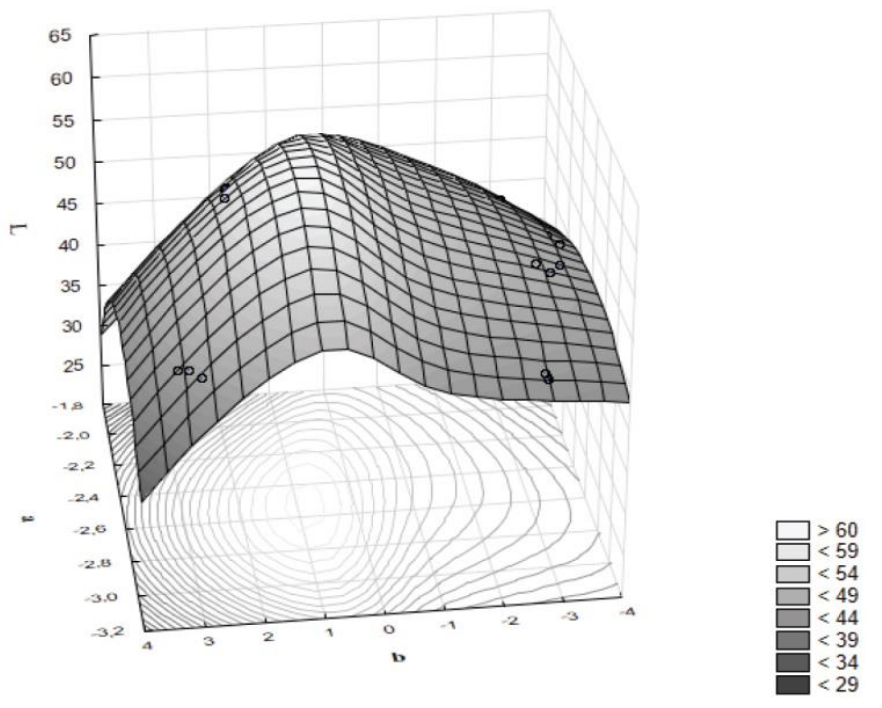

Figure 2. Surface plot of color parameters: $L^{*}$ relative to $b^{*}$ and $a^{*}$.

\subsection{The Rancimat Test}

Oleogels as mixtures, consisting of $95 \% w / w$ of rapeseed oil, containing mainly unsaturated fatty acids [38], are subject to e.g., oxidation process. To determine the rate of oxidative changes occurring in rapeseed oil gels, the Rancimat test was performed (Figure 3). The longest induction time (OSI), $6.8 \mathrm{~h}$, was characterized by the sunflower wax variant. Other oleogels, in particular those containing candelilla wax or white beeswax, obtained OSI not exceeding $5.5 \mathrm{~h}$. This indicates a greater lability of the samples, which may be related to the chemical composition of gelling substances. According to the research of Sindhu Kanya et al. [39], among the free fatty acids, sunflower wax contains the most saturated acids: arachidic (C 20:0) $-47.6 \%$ and behenic (C 22:0) $-25.8 \%$. In the case of beeswax, Tada et al. [40] noted the presence of unsaturated oleic acid (C 18:1) in their chemical composition, which may affect the faster oxidation of BW and BY oleogel samples, compared to the SUN wax variant.

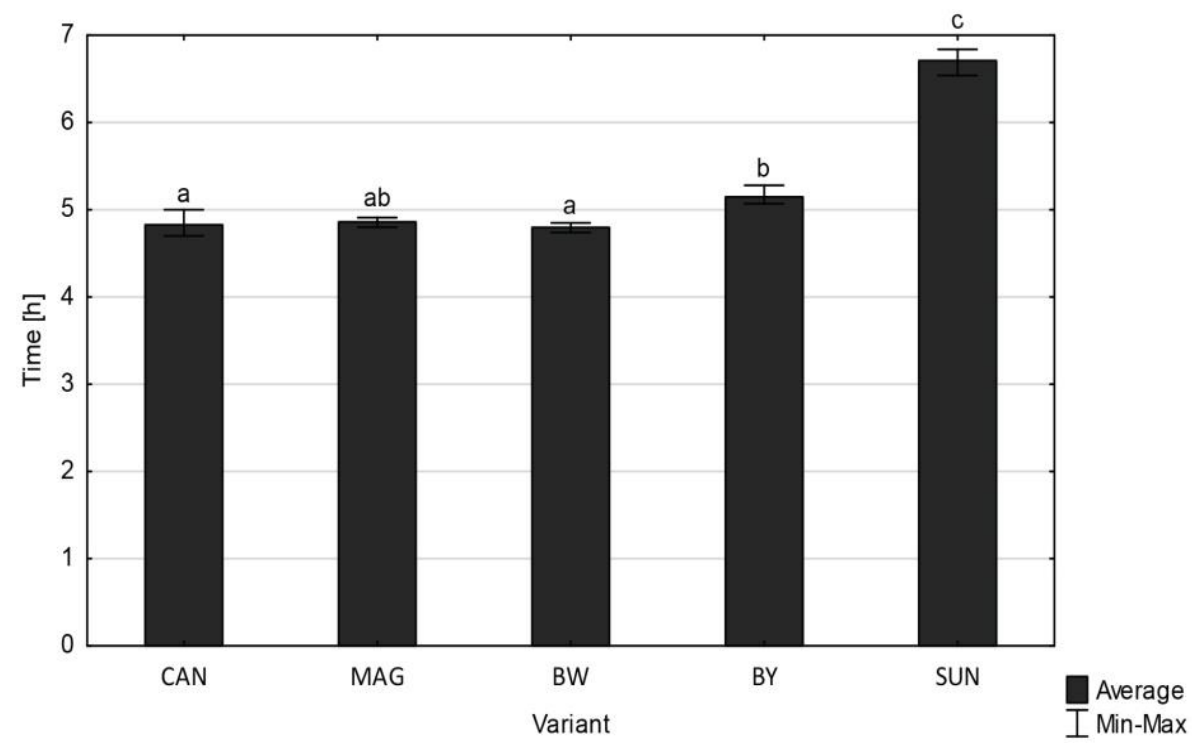

Figure 3. Oleogels induction time; $a, b$, and c describe the homogenous group $(p \leq 0.05)$.

\subsection{Analysis of Microscopic Images}

Both polarized light microscopy and sample observations in the bright field allowed the analysis of the internal structure of the oil gels (Figure 4). Its image depends, among others, on the type 
of gelling substance, temperature, and cooling time of the mixture [41]. The CAN variant had the shortest (on average $8.49 \mathrm{~nm}$ ) and the most regularly distributed crystals compared to other organogels. According to Blake et al. [18], the chemical composition of candelilla wax predominates with hydrocarbons (up to 65\%), including hentriacontane (C31H64) [40]. Other gelling substances, i.e., sunflower wax, monoacylglycerols, yellow beeswax, and white beeswax formed in rapeseed oil thin, much more elongated crystals that were the most visualized using the bright field technique (Figure 4). Their average lengths were $27.41 \mathrm{~nm}, 17.77 \mathrm{~nm}$, and $10.09 \mathrm{~nm}$, respectively (Table 4). According to Hwang et al. [42], esters (ES) are responsible for the morphology of crystals in the form of "needles" in a sample of SUN oleogel. They are the main component of sunflower wax, up to $100 \%$ [18]. It can be assumed that the structure-forming substance that was used was responsible for a similar picture of oleogels with beeswax (Figure 4). In the composition of beeswax, the ES content dominates over the other components [43]. Therefore, it can be said that the esters, which are part of the chemical composition of the above waxes, form "needle" networks of fatty crystals in structured oils. In the case of the variant containing monoacylglycerols, fat clusters were unevenly distributed in the tested sample (Figure 4.2).

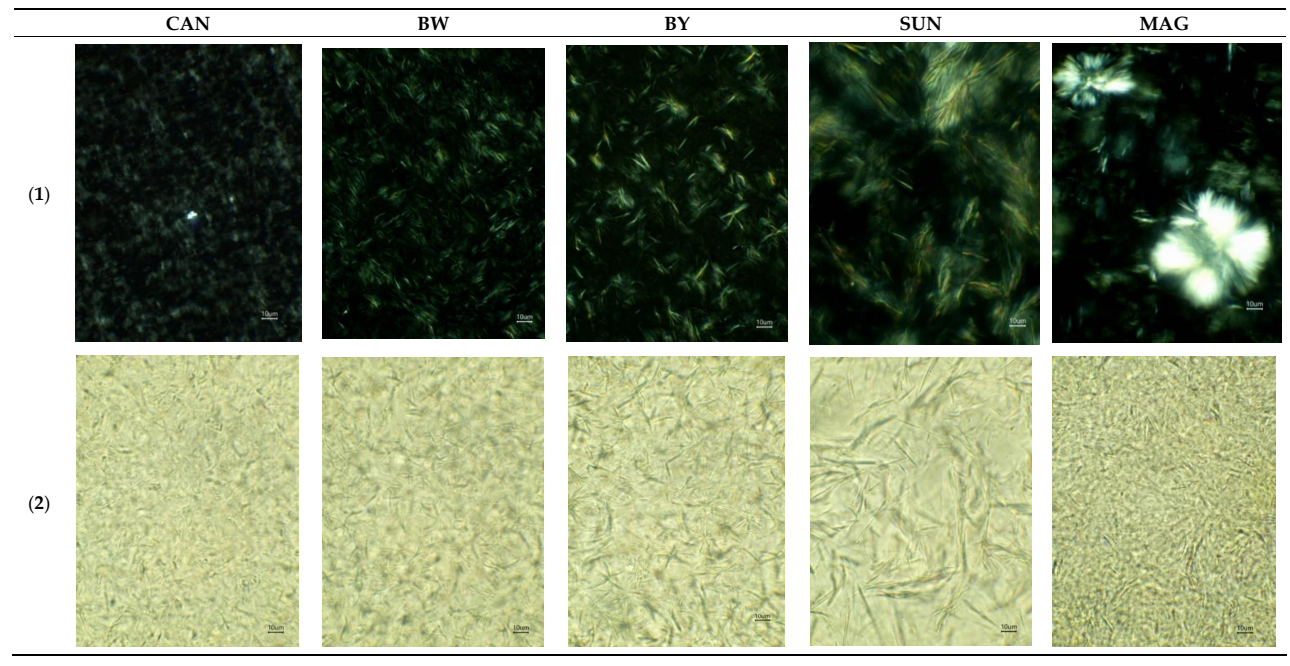

Figure 4. The structure of oleogels in the microscopic image at $600 \times$ magnification, obtained by polarization (images at the top-1) and bright field (images at the bottom-2).

Table 4. Average, minimum (min), and maximum (max) length of oleogels crystals, seeing in polarized microscopy in $600 \times$.

\begin{tabular}{cccc}
\hline Variant of Oleogel & Average $[\mathrm{nm}]$ & Min $[\mathrm{nm}]$ & Max $[\mathrm{nm}]$ \\
\hline CAN & $8.49 \pm 2.53 \mathrm{a}$ & 5.18 & 13.31 \\
MAG & $15.50 \pm 5.19 \mathrm{~b}$ & 9.14 & 23.04 \\
BW & $10.09 \pm 4.02 \mathrm{a}$ & 6.40 & 18.22 \\
BY & $17.77 \pm 8.60 \mathrm{~b}$ & 9.14 & 33.42 \\
SUN & $27.41 \pm 10.42 \mathrm{c}$ & 18.42 & 51.26 \\
\hline
\end{tabular}

$\mathrm{a}, \mathrm{b}, \mathrm{c}-$ the same letters at the second column (Average) are not significantly different $(\alpha=0.05)$.

Analysis of the microscopic image of organogels in polarized light showed the presence of not only individual fat crystals, but also their clusters (Figure 4.1). The largest of these were observed in the monoacylglycerols oleogel. According to Rocha et al. [31], the higher the value of force causing a deformation of the sample, the denser the organogel network. This thesis was confirmed by polarized light images of CAN organogel with the most packed structure (Figure 4) and the highest strength value in the penetration test (Table 2). This observation technique also allowed isolating variants with the most even distribution of crystals in the oil gel. They were organogels with candelilla wax 
and beeswax white. This suggests that the mentioned gelling agents form the most connected network of fat crystals in rapeseed oil-based oleogels, making these systems more stable, compared to the other oleogels. Such a thesis is confirmed by the results of the centrifugation test, which showed the highest stability of CAN and BW variants (Figure 1).

\subsection{Conclusions}

Based on the analysis of the results of oleogels with the $5 \%$ addition of waxes-candelilla (CAN), sunflower (SUN), beeswax white (BW), or yellow (BY) - it was found that these substances have the ability to structure rapeseed oil (OR). The physicochemical properties of OR-based organogels depended on the type of gelling substance used. Considering the highest hardness, stability to centrifugal force, as well as the densest packing of crystals, visible in a microscopic image (at a sample temperature of $20 \pm 2{ }^{\circ} \mathrm{C}$ ), candelilla wax was regarded the most effective structure-forming substance. Therefore, it can be assumed that CAN oleogels could replace, for example, shortenings, which were obtained so far on the basis of solid lipids modified by partial hydrogenation. Such organogels could be used as a raw material for food production, requiring the addition of a stable fat structure and solid consistency, e.g., bakery and pastry products. In turn, the highest oxidative stability (in the Rancimat test) was demonstrated by oleogel with sunflower wax. This result is also very important in the aspect of its potential application, e.g., in food or cosmetics, which during production or storage are exposed to oxidation of fat contained in their composition.

Author Contributions: Conceptualization, M.K. and A.Z.; methodology, M.K. and A.Z.; resources, M.K.; validation, M.K., A.Z., K.M.-L., M.K.; formal analysis, M.K.; data curation A.Z., K.M.-L.; M.K.; writing-original draft preparation, M.K.; writing—review and editing, M.K., A.Z., K.M.-L., M.K.; edited the article for submission, A.Z., K.M.-L., M.K.; visualization, M.K.; supervision A.Z. All authors have read and agreed to the published version of the manuscript.

Funding: This research was funded by Warsaw University of Life Sciences.

Acknowledgments: The authors would like to thank Lasenor Emul (Spain), Kahl GmbH \& Co. KG (Germany) and Norevo GmbH (Germany) for providing free research materials.

Conflicts of Interest: The authors declare no conflict of interest.

\section{References}

1. USDA (United States Department of Agriculture). Oilseeds: World Markets and Trade. Global Market Analysis; Foreign Agricultural Service; United States Department of Agriculture: Washington, DC, USA, 2020; pp. 1-39.

2. Unger, E.H. Commercial processing of canola and rapeseed: Crushing and oil extraction. In Canola and Rapeseed: Production, Chemistry, Nutrition and Processing Technology; Shahidi, F., Ed.; Springer: Boston, MA, USA, 1990; pp. 235-249.

3. Warner, D.; Lewis, K. Evaluation of the Risks of Contaminating Low Erucic Acid Rapeseed with High Erucic Rapeseed and Identification of Mitigation Strategies. Agriculture 2019, 9, 190. [CrossRef]

4. McCarthy, A.; O'Callaghan, Y.C.; O’Brien, N.M. Protein Hydrolysates from Agricultural Crops-Bioactivity and Potential for Functional Food Development. Agriculture 2013, 3, 112-130. [CrossRef]

5. Lin, L.; Allemekinders, H.; Dansby, A.; Campbell, L.; Durance-Tod, S.; Berger, A.; Jones, P.J.H. Evidence of health benefits of canola oil. Nutr. Rev. 2013, 71, 370-385. [CrossRef]

6. Kanwu Study Group; Rasmussen, B.M.; Vessby, B.; Uusitupa, M.; Berglund, L.; Pedersen, E.; Riccardi, G.; Rivellese, A.; Tapsell, L.; Hermansen, K. Effects of dietary saturated, monounsaturated, and n-3 fatty acids on blood pressure in healthy subjects. Am. J. Clin. Nutr. 2006, 83, 221-226. [CrossRef] [PubMed]

7. Mozaffarian, D.; Clarke, R. Quantitative effects on cardiovascular risk factors and coronary heart disease risk of replacing partially hydrogenated vegetable oils with other fats and oils. Eur. J. Clin. Nutr. 2009, 63, S22-S33. [CrossRef] [PubMed]

8. FDA US (Food and Drug Administration). Qualified Health Claims-Qualified Health Claims: Letter of Enforcement Discretion: Unsaturated Fatty Acids from Canola Oil and Reduced Risk of Coronary Heart Disease. 2006 (Docket No. 2006Q-0091). Available online: http://www.fda.gov/Food/LabelingNutrition/ LabelClaims (accessed on 3 May 2020). 
9. Shahbandeh, M. Production Volume of Rapeseed Oil Worldwide from 2012/13 to 2019/20. Statista Farming Report. Available online: https://www.statista.com/statistics/613487/rapeseed-oil-production-volumeworldwide (accessed on 25 April 2020).

10. Jang, A.; Bae, W.; Hwang, H.-S.; Lee, H.G.; Lee, S. Evaluation of canola oil oleogels with candelilla wax as an alternative to shortening in baked goods. Food Chem. 2015, 187, 525-529. [CrossRef] [PubMed]

11. Patel, A.R.; Dewettinck, K. Edible oil structuring: An overview and recent updates. Food Funct. 2016, 7, 20-29. [CrossRef]

12. Callau, M.; Sow-Kébé, K.; Nicolas-Morgantini, L.; Fameau, A.-L. Effect of the ratio between behenyl alcohol and behenic acid on the oleogel properties. J. Colloid Interface Sci. 2020, 560, 874-884. [CrossRef] [PubMed]

13. Delbecq, F.; Nguyen, R.; Van Hecke, E.; Len, C. Design and physicochemical properties of long and stiff fatty low molecular weight oleogelators. J. Mol. Liq. 2019, 295, 111708. [CrossRef]

14. Hwang, H.-S.; Kim, S.; Singh, M.; Moser, J.K.; Liu, S.X. Organogel Formation of Soybean Oil with Waxes. J. Am. Oil Chem. Soc. 2011, 89, 639-647. [CrossRef]

15. Patel, A.R.; Babaahmadi, M.; Lesaffer, A.; Dewettinck, K. Rheological Profiling of Organogels Prepared at Critical Gelling Concentrations of Natural Waxes in a Triacylglycerol Solvent. J. Agric. Food Chem. 2015, 63, 4862-4869. [CrossRef] [PubMed]

16. Giacomozzi, A.; Palla, C.; Carrín, M.E.; Martini, S. Physical Properties of Monoglycerides Oleogels Modified by Concentration, Cooling Rate, and High-Intensity Ultrasound. J. Food Sci. 2019, 84, 2549-2561. [CrossRef] [PubMed]

17. Fu, H.; Lo, Y.M.; Yan, M.; Li, P.; Cao, Y. Characterization of thermo-oxidative behavior of ethylcellulose oleogels. Food Chem. 2020, 305, 125470. [CrossRef] [PubMed]

18. Blake, A.I.; Co, E.D.; Marangoni, A.G. Structure and Physical Properties of Plant Wax Crystal Networks and Their Relationship to Oil Binding Capacity. J. Am. Oil Chem. Soc. 2014, 91, 885-903. [CrossRef]

19. Wijarnprecha, K.; Aryusuk, K.; Santiwattana, P.; Sonwai, S.; Rousseau, D. Structure and rheology of oleogels made from rice bran wax and rice bran oil. Food Res. Int. 2018, 112, 199-208. [CrossRef] [PubMed]

20. Kim, J.Y.; Lim, J.; Lee, J.; Hwang, H.-S.; Lee, S. Utilization of Oleogels as a Replacement for Solid Fat in Aerated Baked Goods: Physicochemical, Rheological, and Tomographic Characterization. J. Food Sci. 2017, 82, 445-452. [CrossRef] [PubMed]

21. Zhao, M.; Lan, Y.; Cui, L.; Monono, E.; Rao, J.; Chen, B. Formation, characterization, and potential food application of rice bran wax oleogels: Expeller-pressed corn germ oil versus refined corn oil. Food Chem. 2020, 309, 125704. [CrossRef]

22. Hughes, N.E.; Marangoni, A.G.; Wright, A.J.; Rogers, M.A.; Rush, J.W. Potential food applications of edible oil organogels. Trends Food Sci. Technol. 2009, 20, 470-480. [CrossRef]

23. Mert, B.; Demirkesen, I. Evaluation of highly unsaturated oleogels as shortening replacer in a short dough product. Food Sci. Technol. 2016, 68, 477-484. [CrossRef]

24. Hwang, H.-S.; Singh, M.; Lee, S. Properties of Cookies Made with Natural Wax-Vegetable Oil Organogels. J. Food Sci. 2016, 81, C1045-C1054. [CrossRef]

25. Franco, D.; Martins, A.J.; López-Pedrouso, M.; Cerqueira, M.A.; Purriños, L.; Pastrana, L.M.; Vicente, A.A.; Zapata, C.; Lorenzo, J.M. Evaluation of linseed oil oleogels to partially replace pork backfat in fermented sausages. J. Sci. Food Agric. 2019, 100, 218-224. [CrossRef] [PubMed]

26. Onacik-Gür, S.; Żbikowska, A. Effect of high-oleic rapeseed oil oleogels on the quality of short-dough biscuits and fat migration. J. Food Sci. Technol. 2019, 57, 1609-1618. [CrossRef]

27. Cegla-Nemirovsky, Y.; Aserin, A.; Garti, N. Oleogels from Glycerol-Based Lyotropic Liquid Crystals: Phase Diagrams and Structural Characterization. J. Am. Oil Chem. Soc. 2015, 92, 439-447. [CrossRef]

28. Bhattacharya, S.; Krishnan-Ghosh, Y. First report of phase selective gelation of oil from oil/water mixtures. Possible implications toward containing oil spills. Chem. Commun. 2001, 185-186. [CrossRef]

29. Abdallah, D.J.; Weiss, R.G. n-Alkanes Geln-Alkanes (and Many Other Organic Liquids). Langmuir 2000, 16, 352-355. [CrossRef]

30. Hwang, H.-S.; Singh, M.; Moser, J.K.; Bakota, E.L.; Liu, S.X. Preparation of Margarines from Organogels of Sunflower Wax and Vegetable Oils. J. Food Sci. 2014, 79, C1926-C1932. [CrossRef]

31. Rocha, J.C.B.; Lopes, J.D.; Mascarenhas, M.C.N.; Arellano, D.B.; Guerreiro, L.M.R.; Cunha, R.L. Thermal and rheological properties of organogels formed by sugarcane or candelilla wax in soybean oil. Food Res. Int. 2013, 50, 318-323. [CrossRef] 
32. Martins, A.J.; Cerqueira, M.A.; Fasolin, L.H.; Cunha, R.L.; Vicente, A. Beeswax organogels: Influence of gelator concentration and oil type in the gelation process. Food Res. Int. 2016, 84, 170-179. [CrossRef]

33. Da Pieve, S.; Calligaris, S.; Co, E.; Nicoli, M.C.; Marangoni, A.G. Shear Nanostructuring of Monoglyceride Organogels. Food Biophys. 2010, 5, 211-217. [CrossRef]

34. Yılmaz, E.; Ögütcü, M.; Yilmaz, E. Properties and Stability of Hazelnut Oil Organogels with Beeswax and Monoglyceride. J. Am. Oil Chem. Soc. 2014, 91, 1007-1017. [CrossRef]

35. Onacik-Gür, S.; Zbikowska, A.; Przybysz, M.; Kowalska, M. Assessment of physical properties of structured oils and palm fat. Mater. Plast. 2017, 54, 800-805. [CrossRef]

36. Mielicki, J. Zarys Wiadomości O Barwie; Fundacja Rozwoju Polskiej Kolorystyki Publisher: Łódź, Poland, 1997.

37. Wrolstad, R.E.; Smith, D.E. Color Analysis. In Food Analysis; Nielsen, S.S., Ed.; Springer: Boston, MA, USA, 2010; pp. 57-586.

38. Codex Alimentarius International Food Standards. Standard for Named Vegetable Oils CXS 210-1999. 1999. Available online: http://www.fao.org/fao-who-codexalimentarius/codex-texts/list-standards/en/ (accessed on 6 June 2020).

39. Kanya, T.C.S.; Rao, L.J.; Sastry, M.C.S. Characterization of wax esters, free fatty alcohols and free fatty acids of crude wax from sunflower seed oil refineries. Food Chem. 2007, 101, 1552-1557. [CrossRef]

40. Tada, A.; Masuda, A.; Sugimoto, N.; Yamagata, K.; Yamazaki, T.; Tanamoto, K. Analysis of constituents of ester-type gum bases used as natural food additives. Shokuhin Eiseigaku Zasshi J. Food Hyg. Soc. Jpn. 2007, 48, 179-185. [CrossRef] [PubMed]

41. Doan, C.D.; Tavernier, I.; Bin Sintang, M.D.; Danthine, S.; Van De Walle, D.; Rimaux, T.; Dewettinck, K. Crystallization and Gelation Behavior of Low- and High Melting Waxes in Rice Bran Oil: A Case-Study on Berry Wax and Sunflower Wax. Food Biophys. 2016, 12, 97-108. [CrossRef]

42. Hwang, H.-S.; Kim, S.; Evans, K.O.; Koga, C.; Lee, Y. Morphology and networks of sunflower wax crystals in soybean oil organogel. Food Struct. 2015, 5, 10-20. [CrossRef]

43. Bonvehí, J.S.; Bermejo, F.O. Detection of adulterated commercial Spanish beeswax. Food Chem. 2012, 132, 642-648. [CrossRef]

(C) 2020 by the authors. Licensee MDPI, Basel, Switzerland. This article is an open access article distributed under the terms and conditions of the Creative Commons Attribution (CC BY) license (http://creativecommons.org/licenses/by/4.0/). 